\title{
L-functions and Random Matrices
}

\author{
J. Brian Conrey \\ American Institute of Mathematics and Oklahoma State University
}

\section{The GUE Conjecture}

\subsection{Introduction}

In 1972 H. L. Montgomery announced a remarkable connection between the distribution of the zeros of the Riemann zeta-function and the distribution of eigenvalues of large random Hermitian matrices. Since then a number of startling developments have occurred making this connection more profound. In particular, random matrix theory has been found to be an extremely useful predictive tool in the theory of L-functions. In this article we will try to explain these recent developments and indicate some directions for future investigations.

\subsection{The Riemann zeta-function}

The Riemann zeta-function is defined by

$$
\zeta(s)=\sum_{n=1}^{\infty} \frac{1}{n^{s}} \quad(s=\sigma+i t, \sigma>1) .
$$

It can also be expressed as a product over primes, the Euler product,

$$
\zeta(s)=\prod_{p}\left(1-\frac{1}{p^{s}}\right)^{-1},
$$

for $\sigma>1$. In 1859 Riemann proved that $\zeta(s)$ extends to a meromorphic function on the whole plane with its only singularity being a simple pole at $s=1$ with residue 1 . He further proved that it has a functional equation relating the value of $\zeta(s)$ with the value of $\zeta(1-s)$,

$$
\zeta(s)=\chi(s) \zeta(1-s)
$$

where $\chi(1-s)=\chi(s)^{-1}=2(2 \pi)^{-s} \Gamma(s) \cos (\pi s / 2)$. He discovered that the distribution of prime numbers is governed by the zeros of $\zeta$. He was led to conjecture that all of the complex zeros $\rho=\beta+i \gamma$ of $\zeta$ have $\beta=1 / 2$. This assertion is the famous Riemann Hypothesis.

We know that $0<\beta<1$ for any complex zero of $\zeta$. Riemann estimated the zero counting function

$$
N(T)=\#\{\rho=\beta+i \gamma: 0<\gamma \leq T\}=\frac{T}{2 \pi} \log \frac{T}{2 \pi e}+O(\log T) .
$$

Thus, at a height $T$ the average spacing between zeros is asymptotic to $2 \pi / \log T$. See [1] for additional background information about $\zeta(s)$. 


\subsection{Background}

Montgomery [M1] was studying gaps between zeros of the Riemann zetafunction in an attempt to prove that the spacings between consecutive zeros can sometimes be less than $1 / 2$ of the average spacing. Such a conclusion would have led to a good effective lower bound for the class number of an imaginary quadratic field. This estimate was not achieved, but through the course of his analysis Montgomery was led to conjecture that

$$
\lim _{T \rightarrow \infty} \frac{1}{N(T)} \sum_{\substack{0 \leq \gamma, \gamma^{\prime} \leq T \\ \frac{2 \pi \alpha}{\log T} \leq \gamma-\gamma^{\prime} \leq \frac{2 \pi \beta}{\log T}}} 1=\int_{\alpha}^{\beta}\left(1-\left(\frac{\sin \pi x}{\pi x}\right)^{2}\right) d x
$$

where $0<\alpha<\beta$ are fixed.

When Montgomery told Freeman Dyson this formula, Dyson responded that the integrand was the pair-correlation function for eigenvalues of large random Hermitian matrices, or more specifically the Gaussian Unitary Ensemble, or GUE.

The GUE is the limit as $N \rightarrow \infty$ of the probability space consisting of $N \times N$ Hermitian matrices $H$ with a probability measure $P(H) d H$ that is invariant under conjugation by any unitary matrix $U$. Here $d H=$ $\prod_{j \leq k} d \Re H_{j k} \prod_{j<k} d \Im H_{j k}$. Mathematical physicists had studied various ensembles since the 1950s in connection with work of Wigner in nuclear physics. Mehta $\mathrm{Me}$ has given a thorough treatment of the development of the subject.

Montgomery went on to conjecture that the $n$-correlation function for zeros of $\zeta$ is the same as that for the GUE; this conjecture came to be known as Montgomery's GUE conjecture.

Odlyzko and Schönhage developed an algorithm that allowed for the simultaneous calculation of many values of $\zeta(1 / 2+i t)$ for $t$ near $T$ in average time $T^{\epsilon}$. This algorithm allowed Odlyzko [0] to do extensive computations of the zeros of $\zeta$ at a height near zero number $10^{20}$; his computations of the pair correlation and nearest neighbor spacing for the zeros of $\zeta$ were amazingly close to those for the GUE. His famous pictures added much credibility to Montgomery's conjecture.

\subsection{Further evidence for GUE}

In 1996 Rudnick and Sarnak [RS] made some interesting progress on the GUE conjecture. To explain their result, number the ordinates of the zeros of $\zeta(s): 0<\gamma_{1} \leq \gamma_{2} \leq \ldots$. Introduce a scaling $\tilde{\gamma}=\gamma \frac{\log \gamma}{2 \pi}$ so that the $\tilde{\gamma}$ have asymptotic mean spacing 1 . Then Sarnak and Rudnick proved that

$$
\lim _{T \rightarrow \infty} \frac{1}{T} \sum_{\substack{\gamma_{j_{1}}, \ldots, \gamma_{j_{n}} \leq T \\ j_{m} \neq j_{n}}} f\left(\tilde{\gamma}_{j_{1}}, \ldots, \tilde{\gamma}_{j_{n}}\right)=\int_{P_{n}} W_{U, n}(\mathbf{x}) f(\mathbf{x}) d \mathbf{x}
$$


where $W_{U, n}(\mathbf{x})=W_{U, n}\left(x_{1}, \ldots, x_{n}\right)$ is the $n$-correlation function for the GUE (see section 5.5) and where $f$ is any function satisfying (1) $f(\mathbf{x}+t(1, \ldots, 1))=f(\mathbf{x})$ for $t \in \mathbb{R} ;(2) f$ is smooth and symmetric in the variables and decays rapidly as $x \rightarrow \infty$ in the hyperplane $P_{n}:=\left\{\left(x_{1}, \ldots, x_{n}\right): \sum_{j=1}^{n} x_{j}=0\right\} ;(3)$ the Fourier transform $\hat{f}(\mathbf{u})$ of $f$ is supported in $\sum_{j=1}^{n}\left|u_{j}\right|<2$. The condition (1) assures that $f$ is a function of the differences of the $\gamma_{j}$.

Thus, the GUE conjecture has been proven for all correlation functions, for a limited class of test functions

\subsection{Other L-functions}

The Riemann zeta-function is the prototype for some extraordinary objects known as arithmetic L-functions. An arithmetic L-function (or Lfunction for short) has many properties in common with the Riemann $\zeta$-function. It has a Dirichlet series

$$
L(s)=\sum_{n=1}^{\infty} a_{n} n^{-s}
$$

it is meromorphic apart from a possible pole at $s=1$; it has a functional equation $\gamma(s) L(s)=\epsilon \overline{\gamma(1-\bar{s}) L(1-\bar{s})}$, where $\epsilon$, the sign, satisfies $|\epsilon|=$ 1. $L(s)$ has an Euler product in which the $p$-th factor is the reciprocal of a polynomial in $p^{-s}$. Moreover, an arithmetic L-function is expected to satisfy the Riemann Hypothesis, that all complex zeros have real part $1 / 2$. (Note that our L-functions are normalized so that the $1 / 2$-line is the line of symmetry for the functional equation. See [B] for precise definitions.) An L-function is called primitive if it is not the product of two L-functions. Primitive L-functions arise from a variety of contexts: from Dirichlet characters, from Mellin transforms of certain cusp forms, from Galois representations, from algebraic varieties, etc. However, it is believed that each primitive arithmetic L-function is associated to a cuspidal automorphic representation of $\mathrm{GL}_{m}$ over a number field.

Rudnick and Sarnak applied their methods to a fairly general Lfunction. They proved the analogue of (6) for any cuspidal automorphic L-function over $\mathbb{Q}$, assuming the Riemann Hypothesis for the L-function, and with obvious changes to reflect the appropriate scaling of the zeros. In particular, the answer did not depend in any way on the distribution of the coefficients of the particular L-function.

The GUE Conjecture is now seen as a universal law governing the distribution of zero spacings for all arithmetic L-functions.

\section{Families}

The realization that the compact classical groups of matrices play a role in the theory of L-functions arose through seminal work of Katz and Sarnak KS1, KS2]. 


\subsection{Function Field Analogues}

Katz and Sarnak investigated the distribution of zeros of function field zeta-functions. Consider a curve $f(x, y)=0$ where $f$ is a polynomial with integer coefficients. The zeta-function for $f$ over a finite field $\mathbb{F}_{q}$ can be obtained in a simple way from the generating function of the numbers $N_{n}$ of points on that curve in the finite field extensions $\mathbb{F}_{q^{n}}, n=1,2, \ldots$ It is known that this zeta-function is a rational function whose numerator is a polynomial with integer coefficients, degree $2 g$ where $g$ is the genus of the curve, and that it satisfies the Riemann Hypothesis that all zeros have modulus $1 / \sqrt{q}$. One can order these zeros on the circle $|z|=\sqrt{q}$ in terms of their angles measured from the positive axis. One then considers statistics of the angles.

Katz and Sarnak proved that, after proper normalization, the $n$ correlation function of these angles, as $g$ and $q$ tend to infinity, is exactly the $n$-correlation of GUE. They also proved that the nearest neighbor, or consecutive spacing, statistic for these angles is the same as that for GUE.

The method of proof involved working with subgroups of $\mathrm{U}(N)$, the group of $N \times N$ unitary matrices with the Haar measure (see sections 5.1 and 5.2). It had been shown by Dyson that the $n$-correlation and the spacing statistics are the same for GUE as for the appropriately scaled limit as $N \rightarrow \infty$ for $U(N)$ with Haar measure. (In the physics literature the limit of $U(N)$ is called the Circular Unitary Ensemble or CUE; see [Me].)

The function field zeta-functions that Katz and Sarnak were working with were characteristic polynomials of matrices from subgroups of $\mathrm{U}(N)$. To deduce their result they applied a theorem of Deligne about the equidistribution of function field zeta-functions among the characteristic polynomials of conjugacy classes in these subgroups.

\section{$2.2 \quad$ Introduction of Families}

Katz and Sarnak investigated the robustness of their theorem. They conjectured that the conclusion remains true under the weaker hypothesis that $q$ is held fixed and $g \rightarrow \infty$. On the other hand, they provided examples of sequences of curves of increasing genus for which the spacing statistic is not the GUE consecutive spacing statistic.

They also considered special families such as (a) curves of the form $y^{2}=f(x)$ with all squarefree, monic $f$, and (b) quadratic twists of curves of the form $y^{2}=x(x-1)(x-t)$; that is $\Delta y^{2}=x(x-1)(x-t)$ where $\Delta$ is a squarefree, monic polynomial over $\mathbb{F}_{q}$. When $q$ and the degree of $\Delta \rightarrow \infty$ they discovered that the $n$-correlation and spacing statistics again matched up with CUE. However, the reason in each case is different depending on the geometric monodromy group. In case (a) they computed that the monodromy group was the symplectic group and in case (b) the orthogonal group. 
We denote by $\operatorname{USp}(N)$ (if $N$ is even) the unitary symplectic matrices, and by $\mathrm{O}(N)$ the orthogonal matrices. These are subgroups of $\mathrm{U}(N)$ and are equipped with their own Haar measure.

Katz and Sarnak proved that the $n$-correlation statistic and the spacing statistic for the limits of $\mathrm{U}(N), \operatorname{USp}(N)$, and $\mathrm{O}(N)$ are all the same as that for GUE.

By contrast, the Circular Orthogonal Ensemble (COE) and the Circular Symplectic Ensemble (CSE), which are well-known ensembles in mathematical physics (see $\mathrm{Me}$ ) have the same underlying symmetry groups as $\mathrm{O}$ and $\mathrm{Sp}$, but have a measure different from the Haar measure. They have different $n$-correlation and spacing statistics than those of GUE and CUE.

From now on we will use the letters $\mathrm{O}, \mathrm{Sp}$, $\mathrm{U}$ when referring to a statistic associated with the limits of $\mathrm{O}(N), \operatorname{USp}(N)$, and $\mathrm{U}(N)$. These statistics are computed for $N \times N$ matrices of the appropriate subgroup of $\mathrm{U}(N)$ with their respective Haar measure and then $N \rightarrow \infty$ with an appropriate scaling limit in order to determine the statistics (see 5.4, 5.5, and 5.6).

Since orthogonal and symplectic matrices have eigenvalues which occur in complex conjugate pairs, it is clear that the eigenvalue 1 plays a special role for these matrices, whereas it does not for unitary matrices. Indeed, there are statistics which differentiate $\mathrm{O}, \mathrm{Sp}$, and U. In particular, the eigenvalue nearest to 1 is such an example. It turns out that this statistic is different for all three symmetry types. More generally the $j$-th eigenvalue nearest to 1 is a statistic that is dependent on the group (for a summary of the four statistics of interest to us, see section 5.3).

Another statistic that is different for all three symmetry types is "level-density". The $n$-level density function is obtained from summing a test function at $n$-distinct eigenvalues. For comparison purposes, note that the $n$-correlation function is obtained by summing a test function of $n$-variables which is a function depending only on the differences of the arguments at $n$ distinct eigenvalues. See sections 5.5 and 5.6 for more on these statistics.

Katz and Sarnak showed that for their special families of function field zeta-functions these new statistics ( $j$-th nearest eigenvalue to 1 and $n$-level density) match with the appropriate statistics from Sp and $\mathrm{O}$, which they had computed.

In general, they found that if they could compute the geometric monodromy group associated with a family of function field zeta-functions, and if that monodromy group was $\mathrm{U}, \mathrm{Sp}$ or $\mathrm{O}$, then all four of the statistics we have been discussing for the function field zeta-functions could be proven to match with the appropriate statistic from U, Sp or O.

Thus, we say that the symmetry type for the family of curves of the form $y^{2}=f(x)$ the symmetry type is $\mathrm{Sp}$; and for the family of quadratic twists of $y^{2}=x(x-1)(x-t)$ the symmetry type is $\mathrm{O}$.

Katz and Sarnak also have examples of families of function field zetafunctions where the symmetry type is $\mathrm{U}$. 


\section{$2.3 \quad$ L-Functions Over Number Fields}

Katz and Sarnak speculated that their results for function field zetafunctions would have implications for L-functions over number fields. Two collections of L-functions which present themselves as natural analogues to the families above are $\left(\mathrm{a}^{\prime}\right)$ the collection of all Dirichlet Lfunctions $L\left(s, \chi_{d}\right)$ where $\chi_{d}(n)$ is a real primitive Dirichlet character with conductor $|d|$ (so that $d$ runs through the set of fundamental discriminants of quadratic number fields) and ( $\left.b^{\prime}\right)$ the collection of L-functions associated with primitive Hecke newforms of a fixed weight. The analogy with the function field zeta-functions is as follows. The zeta-function for a member of the Sp family (a) above is obtained by counting solutions to the equation $y^{2}=f(x)$ over a finite field. These solutions are counted in a field with $p$ elements by the sum $\sum_{a=1}^{p} \chi_{p}(f(a))$ where the real Dirichlet character $\chi_{p}$ modulo $p$ appears. This suggests that the family $\left(\mathrm{a}^{\prime}\right)$ could have a symmetry type Sp. Similarly, the zeta-function of a member of the $\mathrm{O}$ family (b) above is the reciprocal of the $p$-th factor in the Euler product for the L-function of the elliptic curve defined through twists of the equation $y^{2}=x(x-1)(x-t)$, the Legendre family of elliptic curves. These L-functions are known (by the solution of the Taniyama - Shimura conjecture) to be associated to primitive Hecke newforms of weight 2 . Thus, the collection $\left(\mathrm{b}^{\prime}\right)$ could well be a family with symmetry type $\mathrm{O}$.

The first evidence that $\left(\mathrm{a}^{\prime}\right)$ is a family with symmetry type Sp came from Michael Rubinstein's thesis [B]. He computed the lowest lying zero of $L\left(s, \chi_{d}\right)$ and the data matched well with the eigenvalue nearest 1 for symplectic matrices. He examined theoretically the $n$-level density of zeros and showed (for test functions with restrictions on the support of their Fourier transforms) that the $n$-level density functions for the zeros of $L\left(s, \chi_{d}\right)$ are identical with the $n$-level density functions for Sp. Precisely, we index the ordinates of the zeros of $L\left(s, \chi_{d}\right)$ as

$$
0 \leq \gamma_{1}^{(d)} \leq \gamma_{2}^{(d)} \leq \ldots
$$

and scale using

$$
\tilde{\gamma}^{(d)}=\gamma^{(d)} \frac{\log \gamma^{(d)}}{2 \pi} .
$$

Let $D^{*}=\sum_{|d| \leq D} 1$. Then Rubinstein proved that

$$
\frac{1}{D^{*}} \sum_{|d| \leq D} \sum_{\substack{\gamma_{j_{1}}, \ldots, \gamma_{j_{n}} \\ j m \neq \neq j_{n}}} f\left(\tilde{\gamma}_{j_{1}}^{(d)}, \ldots, \tilde{\gamma}_{j_{n}}^{(d)}\right) \rightarrow \int_{\mathbb{R}^{n}} W_{S p, n}(\mathbf{x}) f(\mathbf{x}) d \mathbf{x},
$$

as $D \rightarrow \infty$ where $W_{S p, n}$ is as in section 5.5 , provided that $f$ is a Schwarz function such that the support of $\hat{f}(u)$ is contained in $\sum_{j=1}^{n}\left|u_{j}\right|<1$. $W_{S p, n}$ is called the $n$-level density function for the symplectic group.

Rubinstein also found evidence that another family has a symmetry type $\mathrm{O}$. To describe this family let $\Delta(z)=\sum_{n=1}^{\infty} \tau(n) \mathrm{e}(n z)$ where 
$\tau$ is Ramanujan's tau-function. It is well-known that $\Delta$ is a primitive Hecke newform of weight 12 and level 1 . The family $\left(\mathrm{c}^{\prime}\right)$ is obtained from quadratic twists of the L-function associated with $\Delta$ namely

$$
L\left(\Delta, s, \chi_{d}\right)=\sum_{n=1}^{\infty} \frac{\tau(n) n^{-11 / 2} \chi_{d}(n)}{n^{s}} .
$$

If $d<0$ then this L-function automatically vanishes at $s=1 / 2$ because the associated functional equation occurs with $\epsilon=-1$. Rubinstein computed the lowest zero of $L\left(\Delta, s, \chi_{d}\right)$ for $d>0$ and the lowest zero above the real axis for $L\left(\Delta, s, \chi_{d}\right)$ with $d<0$. At this point, we should mention that an orthogonal matrix has determinant +1 or -1 . So there are actually two symmetry types $\mathrm{O}^{+}$and $\mathrm{O}^{-}$(and the statistics of $\mathrm{O}$ are an average of the statistics of these). Katz and Sarnak had computed the statistics (neighbor spacing, correlations, density, eigenvalue nearest 1) for these two symmetry types as well. Rubinstein found that the lowest lying zero of L-functions in $\left(\mathrm{c}^{\prime}\right)$ with $d>0$ followed $\mathrm{O}^{+}$while the lowest lying zero above the real axis for L-functions with $d<0$ followed $\mathrm{O}^{-}$.

Rubinstein considered more generally the twisting of an arbitrary arithmetic L-function $L(f, s)$ associated with an automorphic form $f$ on $G L_{m}$ by quadratic characters $\chi_{d}$. The results here divide into three cases which have to do with signs of the functional equations. If the L-function $L\left(f, s, \operatorname{sym}^{2}\right)$ associated with the symmetric square of $f$ is entire, the functional equations for $L\left(f, s, \chi_{d}\right)$ will always have $\epsilon=+1$ and the average over $d$ is exactly as above with the $n$-level density function being $W_{S p, n}$. In the case that the symmetric square $L\left(f, s, \mathrm{sym}^{2}\right)$ has a pole (at $s=1)$ then the sign of the functional equation for $L\left(f, s, \chi_{d}\right)$ is +1 for even characters $\chi_{d}$ and -1 for odd characters $\chi_{d}$. Rubinstein averages over these two cases separately and discovers that the first case yields an $n$-level density function $W_{O^{+}, n}$ and in the second case an $n$-level density function $W_{O^{-}, n}$ (see section 5.5). In each of these cases a restriction is placed on the support of the Fourier transform of $f$.

\subsection{The Diagonal Terms}

Rubinstein's work gave impressive confirmation of the theory, but the severe restriction on the support of the Fourier transform is worth investigating. In fact, this restriction occurs right at the place where something interesting is happening with the Fourier transfom of the density function. Returning for a moment to the case of the Riemann zeta-function, we can illustrate this idea.

Montgomery's original theorem involved

$$
F(\alpha, T)=\frac{1}{N(T)} \sum_{0<\gamma, \gamma^{\prime} \leq T} T^{i \alpha\left(\gamma-\gamma^{\prime}\right)} w\left(\gamma-\gamma^{\prime}\right)
$$

Here $w$ is a weight function that concentrates at the origin. (Montgomery used $w(u)=4 /\left(4+u^{2}\right)$.) Assuming the Riemann Hypothesis he showed 
that

$$
F(\alpha, T)=T^{-2 \alpha}(1+o(1))+|\alpha|+o(1)
$$

uniformly for $-1+\epsilon<\alpha<1-\epsilon$ for any $\epsilon>0$.

By the definition of $F$,

$$
\frac{1}{N(T)} \sum_{0<\gamma, \gamma^{\prime} \leq T} r\left(\gamma-\gamma^{\prime}\right) w\left(\gamma-\gamma^{\prime}\right)=\int_{-\infty}^{\infty} \hat{r}(\alpha) F(\alpha, T) d \alpha .
$$

Thus, Montgomery's theorem gives information about the average behavior of differences between the zeros for test functions $r$ with the support of $\hat{r}$ contained in $(-1,1)$. Montgomery went on to conjecture (based on considerations of the behavior of prime pairs) that for $|\alpha|>1$ one has $F(\alpha, T)=1+o(1)$; this assertion implies (1). Thus, $F$ is not differentiable at $\alpha=1$. In the proof of Montgomery's theorem (via the explicit formula and the mean value theorem (23) for Dirichlet polynomials) for $|\alpha|<1$, the main term of $F$ arises from the "diagonal" contributions of the mean square of a Dirichlet polynomial (i.e. the terms $m=n$ in the integral $\int_{0}^{T} a_{m} \overline{a_{n}}(m / n)^{i t} d t$ ). For $\alpha>1$, the off-diagonal terms (i.e. $m \neq n$ ) contribute to the main-term.

A similar situation arises in the work of Rudnick and Sarnak and in the work of Rubinstein. All of the proofs of these theorems are valid only in the range where the diagonal terms dominate.

\subsection{Beyond the Diagonal}

So far we have seen that the theory of families is confirmed by numerical data as well as theoretical data up to the diagonal. Bogolmony and Keating gave a heuristic derivation of all of the GUE conjecture (i.e. all the $n$-level correlations) based on Hardy-Littlewood type conjectures for pairs of primes and pairs of almost primes; this work shows how the off-diagonal terms potentially contribute.

Özlük $\mathrm{Oz}$. proved an analogue for all primitive Dirichlet characters for the pair correlation theorem (12); he obtained a result for $|\alpha|<2$. This was the first example of going beyond the diagonal. See also [OS] and [S2].

It is of great interest that Iwaniec, Luo, and Sarnak [ILS] have succeeded in going beyond the diagonal in several examples which represent three of the symmetry types. They work with the 1-level density functions assuming only that the Riemann Hypothesis holds for all of the L-functions in question. The 1-level density functions may be obtained from $W_{n}$ by taking $n=1$. They are

$$
\begin{gathered}
W(\mathrm{O})(x)=1+\frac{1}{2} \delta_{0}(x), \\
W\left(\mathrm{O}^{+}\right)(x)=1+\frac{\sin 2 \pi x}{2 \pi x},
\end{gathered}
$$




$$
\begin{gathered}
W\left(\mathrm{O}^{-}\right)(x)=1-\frac{\sin 2 \pi x}{2 \pi x}+\delta_{0}(x), \\
W(\mathrm{Sp})(x)=1-\frac{\sin 2 \pi x}{2 \pi x} \\
W(\mathrm{U})(x)=1 .
\end{gathered}
$$

The families considered by Iwaniec, Luo, and Sarnak are related to modular forms. Let $H_{k}^{*}(N)$ denote the set of holomorphic newforms $f$ of weight $k$ and level $N$. Let $H_{k}^{+}(N)$ denote the weight $k$ level $N$ newforms for which the associated L-function has a + in its functional equation, and $H_{k}^{-}(N)$ is the subset of $f$ for which $L(f, s)$ has a - in its functional equation. Let $M^{*}(K, N)$ be the union of the $H^{*}(k, N)$ for $k \leq K$ and similarly define $M^{+}$and $M^{-}$. They consider the low lying zeros of $L(f, s)$ as $f$ varies through one of the $M$-sets. The average spacing for all the zeros of all the $L(f, s)$ with $f \in H^{*}(k, N)$ up to a fixed height $t_{0}$ is asymptotic to $2 \pi / \log \left(k^{2} N\right)$. Let $\phi$ be a test function which is even and rapidly decaying. They proved that if the support of $\hat{\phi}$ is contained in $(-2,2)$, then

$$
\frac{1}{\left|M^{*}(K, N)\right|} \sum_{\substack{f \in M^{*}(K, N) \\ \gamma_{f}}} \phi\left(\frac{\gamma_{f} \log k^{2} N}{2 \pi}\right) \rightarrow \int_{-\infty}^{\infty} \phi(x) W(\mathrm{O})(x) d x
$$

as $K N \rightarrow \infty$. Similar statements hold with $M^{*}$ replaced by $M^{+}$and $M^{-}$and $\mathrm{O}$ replaced by $\mathrm{O}^{+}$and $\mathrm{O}^{-}$.

It should be pointed out that the Fourier transforms of the density functions $W(\mathrm{O})(x), W\left(\mathrm{O}^{+}\right)(x)$, and $W\left(\mathrm{O}^{-}\right)(x)$ all agree in the diagonal range; so it is only when one goes beyond the diagonal that the distinguishing features of these three symmetry types becomes apparent.

Iwaniec, Luo, and Sarnak also consider the symmetric square Lfunctions of the $f \in M^{*}$ and verify that the above statements hold with symmetry type Sp and the support of $\hat{\phi}$ in $(-3 / 2,3 / 2)$. Also, the average zero spacing is $2 \pi / \log \left(k^{2} N^{2}\right)$ so $N$ should be replaced by $N^{2}$ in the argument of $\phi$ in the left hand side of (20).

Thus, the theoretical and numerical evidence that the zeros of families of L-functions depend on the symmetry type of the family is pretty convincing.

\section{Moments}

So far we have seen that the eigenvalues of matrices from unitary groups are excellent models for zeros of families of L-functions. Now we want to take the matrix models a significant step further and argue that the characteristic polynomials of these matrices on average reveal very important features of the value distribution of the L-functions in the family. 


\subsection{Moments of the Riemann zeta-function}

We first look at the situation of the Riemann zeta-function and its moments.

To give some background, we cite the theorem of Hardy and Littlewood:

$$
\frac{1}{T} \int_{0}^{T}\left|\zeta\left(\frac{1}{2}+i t\right)\right|^{2} d t \sim \log T
$$

and the theorem of Ingham:

$$
\frac{1}{T} \int_{0}^{T}\left|\zeta\left(\frac{1}{2}+i t\right)\right|^{4} d t \sim \frac{1}{2 \pi^{2}} \log ^{4} T .
$$

The asymptotics of no other moments (apart from the trivial 0-th moment) are known. In general it has been conjectured that

$$
\frac{1}{T} \int_{0}^{T}\left|\zeta\left(\frac{1}{2}+i t\right)\right|^{2 k} d t \sim c_{k} \log ^{k^{2}} T .
$$

The basic tools for investigating mean-values in $t$-aspect are the mean value theorem for Dirichlet polynomials (due to Montgomery and Vaughan):

$$
\int_{0}^{T}\left|\sum_{n \leq N} a_{n} n^{i t}\right|^{2} d t=\sum_{n \leq N}(T+O(n))\left|a_{n}\right|^{2}
$$

and some sort of formula expressing the function in question in terms of Dirichlet polynomials (such as an approximate functional equation) such as

$$
\zeta(s)^{k}=\sum_{n \leq \tau^{k}} \frac{d_{k}(n)}{n^{s}}+\chi(s)^{k} \sum_{n \leq \tau^{k}} \frac{d_{k}(n)}{n^{1-s}}+E(s),
$$

where $E(s)$ should be small on average, $s=1 / 2+i t, \tau=\sqrt{t /(2 \pi)}, \chi(s)$ is the factor from the functional equation (3), and where

$$
\zeta(s)^{k}=\sum_{n=1}^{\infty} \frac{d_{k}(n)}{n^{s}} \quad(\sigma>1)
$$

so that $\zeta(s)^{k}$ is the generating function for $d_{k}(n)$. Note that the meanvalue theorem for Dirichlet polynomials detects only diagonal contributions.

Conrey and Ghosh CG2 gave the moment conjecture a more precise form, namely that there should be a factorization

$$
c_{k}=\frac{g_{k} a_{k}}{\Gamma\left(1+k^{2}\right)}
$$

where

$$
a_{k}=\prod_{p}\left(1-\frac{1}{p}\right)^{k^{2}} \sum_{j=0}^{\infty} \frac{d_{k}\left(p^{j}\right)^{2}}{p^{j}}
$$


is an arithmetic factor and $g_{k}$, a geometric factor, should be an integer. Note that by the mean-value theorem for Dirichlet polynomials it is not difficult to show that

$$
\frac{1}{T} \int_{0}^{T}\left|\sum_{n \leq x} \frac{d_{k}(n)}{N^{1 / 2+i t}}\right|^{2} d t \sim \frac{a_{k}(\log x)^{k^{2}}}{\Gamma\left(1+k^{2}\right)}
$$

provided that $x=o(T)$. Thus, an interpretation of $g_{k}$ is

$$
g_{k}=\lim _{T \rightarrow \infty} \frac{\int_{0}^{T}\left|\zeta^{k}\left(\frac{1}{2}+i t\right)\right|^{2} d t}{\int_{0}^{T} \mid \sum_{n \leq T} \frac{d_{k}(n)}{\left.n^{1 / 2+i t}\right|^{2}} d t}
$$

assuming that the limit exists, so that $g_{k}$ represents the 'number' of Dirichlet polynomial approximations to $\zeta(s)^{k}$ of length $T$ required to measure the mean square of $\zeta(s)^{k}$.

In this notation, the result of Hardy and Littlewood is that $g_{1}=1$ and Ingham's result is that $g_{2}=2$.

These results can be obtained essentially from the mean-value theorem for Dirichlet polynomials. To go beyond the fourth moment requires taking into account off-diagonal contributions. Goldston and Gonek GG] describe a precise way to transform information about coefficient correlations $\sum_{n \leq x} a(n) a(n+r)$ into a formula for the mean square of a long Dirichlet polynomial $\sum_{n \leq x} a(n) n^{-s}$ where $x$ is bigger than the length of integration.

Using Dirichlet polynomial techniques Conrey and Ghosh CG1 conjectured that $g_{3}=42$ and Conrey and Gonek CGo] conjectured that $g_{4}=24024$. Meanwhile, Keating and Snaith KeSn1 computed the moments of characteristic polynomials of matrices in $U(N)$ and found that for any real $x$ and any complex number $s$,

$$
M_{U, N}(s)=\int_{U(N)}|\operatorname{det}(A-I \exp (-i x))|^{2 s} d A=\prod_{j=1}^{N} \frac{\Gamma(j) \Gamma(j+2 s)}{\Gamma(j+s)^{2}},
$$

where $d A$ denotes the Haar measure for the group $U(N)$ of $N \times N$ unitary matrices. To do this calculation, they made use of Weyl's formula for the Haar measure (see section 5.3) and Selberg's integral (see section 5.6). They also showed that

$$
\lim _{N \rightarrow \infty} \frac{M_{N}(s)}{N^{s^{2}}}=\frac{G(1+s)^{2}}{G(1+2 s)},
$$

where $G(s)$ is Barnes' double Gamma-function which satisfies $G(1)=1$ and $G(z+1)=\Gamma(z) G(z)$. Note that for $s=k$ an integer,

$$
\frac{G(1+k)^{2}}{G(1+2 k)}=\prod_{j=0}^{k-1} \frac{j !}{(j+n) !}
$$


For $k=1,2,3$ the above is $1 / 1$ !,2/4!, 42/9! in agreement with the theorems of Hardy and Littlewood, and Ingham and the conjecture of Conrey and Ghosh. Keating and Snaith argued that one should thus model the moments of the zeta-function from 0 to $T$ by moments of characteristic polynomials of unitary matrices of size $N \sim \log T$. (More precisely, one should take $N$ to be the integer nearest to $\log \frac{T}{2 \pi}$ ). They then conjectured that

$$
g_{k}=k^{2} ! \prod_{j=0}^{k-1} \frac{j !}{(j+k) !}
$$

for integer $k$. The initial public announcements of the conjectures of Conrey and Gonek (that $g_{4}=24024$ ) and of Keating and Snaith ( $g_{k}$ for all real $k \geq-1 / 2$ ) occurred at the Vienna conference on the Riemann Hypothesis only moments after it was checked that the Keating and Snaith conjecture does indeed predict that $g_{4}=24024$.

\subsection{Moments of L-functions at $1 / 2$}

Subsequently, Conrey and Farmer CF analyzed known results for moments of L-functions at $1 / 2$ and made a general conjecture. (These moments had been considered by a number of authors; see especially $[\mathrm{GV}$.) The conjecture has the shape

$$
\frac{1}{X^{*}} \sum_{\substack{f \in \mathcal{F} \\ c(f) \leq X}} L(f, 1 / 2)^{k} \sim \frac{g_{k} a_{k}}{\Gamma(1+q(k))}(\log X)^{q(k)}
$$

for some $a_{k}, g_{k}$, and $q(k)$ where $\mathcal{F}$ is a family of $f$ parametrized by the conductor $c(f)$, and $X^{*}=\sum_{c(f) \leq X}$. The observations of Conrey and Farmer were that $g_{k}$ and $q(k)$ depend only on the symmetry type of the family, and that $a_{k}$ depends on the family itself, but is explicitly computable in any specific case. Thus, the conjecture is that $q(k)=k^{2}$ for a unitary family, $q(k)=k(k+1) / 2$ for a symplectic family, and $q(k)=$ $k(k-1) / 2$ for an orthogonal family. The values of $g_{k}$ were left unspecified, but were then predicted as before from random matrix theory by Keating and Snaith [KeSn2] and independently by Brezin and Hikami [BH] by computing moments of characteristic polynomials of matrices from $\mathrm{O}(N)$ and from $\mathrm{USp}(2 N)$. Each is a quotient of products of Gamma-functions.

Thus, for the family of Dirichlet L-functions $L\left(s, \chi_{d}\right)$ with a real primitive Dirichlet character $\chi_{d}$ modulo $d$ we have the following results $\left(D^{*}=\sum_{|d| \leq D} 1\right):$ Jutila $[\mathrm{J}]$ proved that

$$
\frac{1}{D^{*}} \sum_{|d| \leq D} L\left(\frac{1}{2}, \chi_{d}\right) \sim a_{1} \log \left(D^{\frac{1}{2}}\right)
$$

and

$$
\frac{1}{D^{*}} \sum_{|d| \leq D} L^{2}\left(\frac{1}{2}, \chi_{d}\right) \sim 2 \frac{a_{2} \log ^{3}\left(D^{\frac{1}{2}}\right)}{3 !}
$$


Soundararajan So1] showed that

$$
\frac{1}{D^{*}} \sum_{|d| \leq D} L^{3}\left(\frac{1}{2}, \chi_{d}\right) \sim 16 \frac{a_{3} \log ^{6}\left(D^{\frac{1}{2}}\right)}{6 !}
$$

and conjectured that

$$
\frac{1}{D^{*}} \sum_{|d| \leq D} L^{4}\left(\frac{1}{2}, \chi_{d}\right) \sim 768 \frac{a_{4} \log ^{10}\left(D^{\frac{1}{2}}\right)}{10 !} .
$$

The general conjecture coming from random matrix theory (see section 5.7), which agrees with the above, is:

$$
\frac{1}{D^{*}} \sum_{|d| \leq D} L^{k}\left(\frac{1}{2}, \chi_{d}\right) \sim \prod_{\ell=1}^{k} \frac{\ell !}{2 \ell !} a_{k} \log ^{k(k+1) / 2}(D)
$$

where

$$
a_{k}=\prod_{p} \frac{\left(1-\frac{1}{p}\right)^{\frac{k(k+1)}{2}}}{\left(1+\frac{1}{p}\right)}\left(\frac{\left(1-\frac{1}{\sqrt{p}}\right)^{-k}+\left(1+\frac{1}{\sqrt{p}}\right)^{-k}}{2}+\frac{1}{p}\right) .
$$

An example of an orthogonal family where several moments are known arises from $\mathcal{F}_{q}$ the set of primitive cusp forms of weight 2 and level $q$ ( $q$ prime). Then, from results of Duke [D], Duke, Friedlander, and Iwaniec [DF], Iwaniec and Sarnak [IS1, and Kowalski, Michel, and VanderKam [KMV1] and KMV2], we have

$$
\begin{gathered}
\frac{1}{\left|\mathcal{F}_{q}\right|} \sum_{f \in \mathcal{F}_{q}} L(1 / 2, f) \sim a_{1} \\
\frac{1}{\left|\mathcal{F}_{q}\right|} \sum_{f \in \mathcal{F}_{q}} L^{2}(1 / 2, f) \sim 2 a_{2} \log q^{\frac{1}{2}} \\
\frac{1}{\left|\mathcal{F}_{q}\right|} \sum_{f \in \mathcal{F}_{q}} L^{3}(1 / 2, f) \sim 8 a_{3} \frac{\log ^{3} q^{\frac{1}{2}}}{3 !} \\
\frac{1}{\left|\mathcal{F}_{q}\right|} \sum_{f \in \mathcal{F}_{q}} L^{4}(1 / 2, f) \sim 128 a_{4} \frac{\log ^{6} q^{\frac{1}{2}}}{6 !}
\end{gathered}
$$

where $a_{1}=\zeta(2)$,

$$
\begin{gathered}
a_{2}=\zeta(2)^{2} \prod_{p}\left(1+\frac{1}{p^{2}}\right) \\
a_{3}=\zeta(2)^{3} \prod_{p}\left(1-\frac{1}{p}\right)\left(1+\frac{1}{p}+\frac{4}{p^{2}}+\frac{1}{p^{3}}+\frac{1}{p^{4}}\right) \\
a_{4}=\zeta(2)^{5} \prod_{p}\left(1-\frac{1}{p}\right)^{3}\left(1+\frac{3}{p}+\frac{11}{p^{2}}+\frac{10}{p^{3}}+\frac{11}{p^{4}}+\frac{3}{p^{5}}+\frac{1}{p^{6}}\right)
\end{gathered}
$$


We have not found a simple expression for $a_{k}$, though it can be determined explicitly for each $k$. As before, a general conjecture is:

$$
\frac{1}{\left|\mathcal{F}_{q}\right|} \sum_{f \in \mathcal{F}_{q}} L^{k}(1 / 2, f) \sim 2^{k-1} \prod_{\ell=1}^{k-1} \frac{\ell !}{2 \ell !} a_{k} \log ^{k(k-1) / 2} q .
$$

We believe that in formulas for moments of L-functions over a family the power of the $\log$ of the conductor and the value of $g_{k}$ should only depend on the symmetry type of the family and that the value of $a_{k}$ will depend on the family but can always be determined explicitly.

\section{Further directions}

In this section we mention some questions where further research is desirable.

\subsection{Full moment conjecture}

What are the lower order terms in the moment formulae for $|\zeta(1 / 2+i t)|^{2 k}$ and for $L(1 / 2)^{k}$ ? These are known in a few instances (see [I] ], [] for the second and fourth moments of $\zeta(s)$ ) but not in general. The difficulty is that random matrix theory does not "see" the contribution of the arithmetic factor $a_{k}$. Lower order terms will likely involve a mix of derivatives of $a_{k}$ and secondary terms from the moments of the characteristic polynomials of matrices. In general, a better understanding of how $\zeta(s)$ is modeled by a characteristic polynomial of a certain type of matrix is needed; how do the primes come into play? Perhaps we should think of $\zeta(1 / 2+i t)$ as a partial Hadamard product over zeros multiplied by a partial Euler product. Perhaps these two parts behave independently, and the Hadamard product part can be modeled by random matrix theory.

\subsection{Distribution of Values}

Keating and Snaith compute explicit formulas for the $s$-th moment of the characteristic polynomials of matrices from $\mathrm{O}(N), \mathrm{Sp}(N)$, and $\mathrm{U}(N)$. Consequently the value distributions for these characteristic polynomials can be explicitly computed; they involve the Fourier transform of the $s$-th moment. Preliminary investigations indicate that there is a good fit between the random matrix formulae and numerical data. One particularly interesting feature of this investigation involves the understanding of zeros which occur exactly at $1 / 2$. These seem to occur only for Lfunctions in an orthogonal family. If the sign of the functional equation is -1 , there is automatically a zero at $1 / 2$. The interesting situation is when the sign is + and there is still a zero; for example if $\mathrm{E}$ is an elliptic curve defined over $\mathbb{Q}$ then examination of the distribution of values of $\mathrm{O}^{+}$suggest that twists $L\left(E, s, \chi_{p}\right)$ of the L-function by quadratic characters seem to vanish for about $X^{3 / 4}(\log X)^{-5 / 8}$ values of $|p|<X$ with $\operatorname{sign}+1$ in the functional equation. 


\subsection{Extreme Values}

How large is the maximum value of $|\zeta(1 / 2+i t)|$ for $T<t<2 T$ ? It is known that the Riemann Hypothesis implies that the maximum is at most $\exp (c \log T / \log \log T)$ for some $c>0$. It is also known that the maximum gets as big as $\exp \left(c_{1}(\log T / \log \log T)^{1 / 2}\right)$ for a sequence of $T \rightarrow \infty$ for some $c_{1}>0$. It has been conjectured that the smaller bound (the one that is known to occur) is closer to the truth. However, the new conjectures about moments suggest that it may be the larger.

This question has a number of equivalent and analogous (for L-values) formulations. How big can $S(T):=\frac{1}{\pi} \arg \zeta(1 / 2+i T)$ be? Assuming the Riemann Hypothesis, it is known that $S(T) \ll \log T / \log \log T$ but that infinitely often it is bigger than $c(\log T / \log \log T)^{1 / 2}$ for some $c>0$ Which is closer to the truth? What is the maximum size of the class number of an imaginary quadratic field (as a function of the discriminant) (see Sh] for a discussion and numerical investigation.)? How big can the least quadratic non-residue of a given prime $p$ be $\left(\log p\right.$ or $(\log p)^{2-\epsilon}$ ? See [M2] for a discussion of this question? What is the maximal order of vanishing of an L-function at $1 / 2$ ? In terms of the conductor $N$, can it be as big as $\log N / \log \log N$ or is it at most the square root of that, or something entirely different? All of these questions are related, at least by analogy, and they may all have similar answers. It would be interesting and surprising if in each case it is the larger bound which is closer to the truth.

\subsection{Zeros of $\zeta^{\prime}(s)$}

Can one use random matrix theory to predict the horizontal distribution of the real parts of the zeros of $\zeta^{\prime}$ ? It is known that the Riemann Hypothesis is equivalent to the assertion that each non-real zero of $\zeta^{\prime}(s)$ has real part greater than or equal to $1 / 2$. Moreover, if such a zero has real part $1 / 2$, then it is also a zero of $\zeta(s)$ (and so a multiple zero of $\zeta(s)$ ). These assertions are the point of departure for Levinson's work on zeros of the Riemann zeta-function on the critical line. It would be interesting to know the horizontal distribution of these zeros; in particular what proportion of them with ordinates between $T$ and $2 T$ are within $a / \log T$ of the $1 / 2$-line?

In a similar vein, the Riemann $\xi$-function is real on the $1 / 2$-line and has all of its zeros there (assuming the Riemann Hypothesis). It is an entire function of order 1 ; because of its functional equation, $\xi(1 / 2+i \sqrt{z})$ is an entire function of order 1/2. It follows that the Riemann Hypothesis implies that all zeros of $\xi^{\prime}(s)$ are on the 1/2-line. Assuming this to be true, one can ask about the vertical distribution of zeros of $\xi^{\prime}(s)$, and more generally of $\xi^{(m)}(s)$. It seems that the zeros of higher derivatives will become more and more regularly spaced; can these distributions be expressed in a simple way using random matrix theory? 


\subsection{Long Mollifiers, Local Integrals, and GUE}

David Farmer [F1], [F2 has made two very interesting conjectures having to do with $\zeta(s)$. The first is a conjecture about the mean square of $\zeta(1 / 2+$ $i t$ ) times an arbitrarily long mollifier. A mollifier is a Dirichlet polynomial with coefficients equal to the Möbius $\mu$-function times a smooth function. The length of the mollifier is the length of the Dirichlet polynomial. He has also conjectured that

$$
\frac{1}{T} \int_{0}^{T} \frac{\zeta(s+u) \zeta(1-s+v)}{\zeta(s+a) \zeta(1-s+b)} d t \sim 1+\frac{(u-a)(v-b)}{(u+v)(a+b)}\left(1-T^{-(u+v)}\right) .
$$

where $a, b, u, v$ are complex numbers with positive real part, and $s=$ $1 / 2+i t$. These two conjectures are essentially equivalent and imply certain parts of the GUE conjecture. It would be interesting to generalize these and relate them to the full GUE conjecture.

\section{Appendices}

\subsection{The Classical Groups}

- The unitary group $\mathrm{U}(N)$ is the group of $N \times N$ matrices $U$ with entries in $\mathbb{C}$ for which $U U^{*}=I$ where $U^{*}$ denotes the conjugate transpose of $U$, i.e. if $U=\left(u_{i, j}\right)$, then $U^{*}=\left(\overline{u_{j, i}}\right)$.

- The orthogonal group $\mathrm{O}(N)$ is the subgroup of $\mathrm{U}(N)$ consisting of matrices with real entries.

- The special orthogonal group $\mathrm{SO}(N)$. This is the subgroup of $\mathrm{O}(N)$ consisting of matrices with determinant $1 . \mathrm{SO}(2 N)$ leads to the symmetry type we have called $\mathrm{O}^{+}$and $\mathrm{SO}(2 N+1)$ leads to the symmetry type we call $\mathrm{O}^{-}$.

- The symplectic group $\mathrm{USp}(2 N)$ is the subgroup of $\mathrm{U}(2 N)$ of matrices $U$ for which $U Z U^{t}=Z$ where $U^{t}$ denotes the transpose of $U$ and

$$
Z=\left(\begin{array}{cc}
0 & I_{N} \\
-I_{N} & 0
\end{array}\right)
$$

\subsection{The Weyl Integration Formula}

The $N \times N$ unitary matrices can be parametrized by their $N$ eigenvalues on the unit circle. Any configuration of $N$ points on the unit circle corresponds to a conjugacy class of $\mathrm{U}(N)$. If $f(A)=f\left(\theta_{1}, \ldots \theta_{N}\right)$ is a symmetric function of $N$ variables, then Weyl's formula [W] gives

$$
\int_{U(N)} f(A) d A=\frac{1}{N !} \int_{[0,1]^{N}} f(\theta) \prod_{1 \leq j<k \leq N}\left|e\left(\theta_{j}\right)-e\left(\theta_{k}\right)\right|^{2} d \theta_{1} \ldots d \theta_{N}
$$


where $d A$ is the Haar measure. Similarly, on $\operatorname{Sp}(2 N)$ and $\operatorname{SO}(2 N)$ we have respectively

$$
\begin{gathered}
d A=\frac{2^{N^{2}}}{N !} \prod_{j<k}\left(\cos \pi \theta_{j}-\cos \pi \theta_{k}\right)^{2} \prod_{j=1}^{N} \sin ^{2} \pi \theta_{j} \prod_{j=1}^{N} d \theta_{j} \\
d A=\frac{2^{(N-1)^{2}}}{N !} \prod_{i<j}\left(\cos \pi \theta_{j}-\cos \pi \theta_{k}\right)^{2} \prod_{j=1}^{N} d \theta_{j} .
\end{gathered}
$$

\subsection{Four Statistics}

Suppose we have a sequence $\mathcal{T}$ of $N$-tuples of numbers $T_{N}=\left\{t_{1}, t_{2}, \ldots, t_{N}\right\}$ where $t_{1} \leq t_{2}<\ldots<t_{N}$ such that for each set the average spacing $t_{j+1}-t_{j}$ is asymptotically 1 . We write $t_{i, N}$ in place of $t_{i}$ if we need to indicate that $t_{i} \in T_{N}$.

- The $n$-level density of $\mathcal{T}$ is $W(\mathbf{x})=W\left(x_{1}, \ldots x_{n}\right)$ means that

$$
\lim _{N \rightarrow \infty} \sum_{\substack{\left(i_{1}, \ldots i_{n}\right), i_{j} \leq N \\ i_{j} \neq i_{k}}} f\left(t_{i_{1}}, \ldots t_{i_{n}}\right)=\int_{\mathbb{R}^{n}} f(\mathbf{x}) W(\mathbf{x}) d \mathbf{x} .
$$

for a Schwarz-class $f$. The sum is over $n$-tuples with distinct entries.

- The $j$-th lowest zero density is $\nu_{j}(x)$ means that for a test function $f$

$$
\lim _{N \rightarrow \infty} \frac{1}{N} \sum_{n \leq N} f\left(t_{j, n}\right)=\int_{0}^{\infty} f(x) \nu_{j}(x) d x .
$$

- The consecutive spacing density is $\mu(x)$ means that for a test function $f(x)$ we have

$$
\lim _{N \rightarrow \infty} \frac{1}{N} \sum_{i \leq N-1} f\left(t_{i+1}-t_{i}\right)=\int_{0}^{\infty} f(x) \mu(x) d x .
$$

- The $n$-correlation density is $V\left(x_{1}, \ldots, x_{n}\right)$ means that for test functions $f$ that are symmetric in all of the variables, depend only on the differences of the variables (i.e. $f\left(x_{1}+u, \ldots, x_{n}+u\right)=$ $f\left(x_{1}, \ldots, x_{n}\right)$ for all $\left.u\right)$, and are rapidly decaying on the hyperplane $P_{n}:\left\{\left(x_{1}, \ldots, x_{n}: \sum x_{i}=0\right\}\right.$, we have, as $N \rightarrow \infty$,

$$
\frac{1}{N} \sum_{\substack{t_{1}, \ldots, t_{n} \in T_{N} \\ i_{j} \neq i_{k}}} f\left(t_{1}, \ldots t_{n}\right) \rightarrow \int_{P_{n}} f(\mathbf{x}) V(\mathbf{x}) d x_{1} \ldots d x_{n-1}
$$

as $N \rightarrow \infty$. The spacing and $n$-correlation densities are universal, i.e. the same for each of $\mathrm{O}, \mathrm{Sp}$, and $\mathrm{U}$, whereas the $n$-level and $j$-th lowest zero densities depend on the symmetry type. 


\subsection{Gaudin's Lemma}

Associated to each $N \times N$ unitary matrix $A$ are its $N$ eigenvalues e $\left(\theta_{j}\right)$ where $0 \leq \theta_{1} \leq \ldots \leq \theta_{N} \leq 1$. We integrate a function $F(A)$ over $\mathrm{U}(N)$ by parametrizing the group by the $\theta_{i}$ and using Weyl's formula to convert the integral into an $N$-fold integral over the $\theta_{i}$.

Often one wants to integrate with respect to Haar measure over $\mathrm{U}(N)$ a function $F(A)=\tilde{f}(A)=\tilde{f}\left(\theta_{1}, \ldots, \theta_{N}\right)$ of $N$ variables that is "lifted" from a function $f$ of $n$ variables:

$$
\tilde{f}\left(\theta_{1}, \ldots, \theta_{N}\right)=\sum_{\substack{\left.i_{1}, \ldots, i_{n}\right) \\ i_{j} \neq i_{k}}} f\left(\theta_{i_{1}}, \ldots, \theta_{i_{n}}\right)
$$

where the sum is over all possible $n$-tuples $\left(i_{1}, \ldots, i_{n}\right)$ of distinct integers between 1 and $N$. Gaudin's lemma gives a simplification of this computation from an $N$-fold integral to an $n$-fold integral. The Haar measure (see section 5.2) at the matrix $A$ can be expressed as

$$
d A=\frac{1}{N !} \prod_{1 \leq j<k \leq N}\left|e\left(\theta_{j}\right)-e\left(\theta_{k}\right)\right|^{2} d \theta_{1} \ldots d \theta_{N} .
$$

The product here is the square of the absolute value of the $N \times N$ Vandermonde determinant with $j, k$ entry e $\left(\theta_{k}\right)^{j-1}=\mathrm{e}\left((j-1) \theta_{k}\right)$. It is also the $N \times N$ determinant of the matrix with $j, k$ entry $J_{N}\left(\theta_{j}-\theta_{k}\right)$ where

$$
J_{N}(\theta)=\sum_{m=0}^{N-1} \mathrm{e}(m \theta)=\mathrm{e}((N-1) \theta / 2) \frac{\sin \pi N \theta}{\sin \pi \theta} .
$$

Thus,

$$
\int_{U(N)} \tilde{f}(A) d A=\int_{[0,1]^{N}} \tilde{f}\left(\theta_{1}, \ldots, \theta_{N}\right) \frac{1}{N !} \operatorname{det}_{N \times N} J_{N}\left(\theta_{j}-\theta_{k}\right) \prod_{j=1}^{N} d \theta_{j} .
$$

Then, Gaudin's lemma asserts the equality

$$
\int_{U(N)} \tilde{f}(A) d A=\int_{[0,1]^{n}} f\left(\theta_{1}, \ldots, \theta_{n}\right) \frac{1}{n !} \operatorname{det}_{n \times n} J_{N}\left(\theta_{j}-\theta_{k}\right) \prod_{j=1}^{n} d \theta_{j} .
$$

This principle works for all of the subgroups of $\mathrm{U}(N)$ under consideration here as well. (See [KS] section 5.1 for a general statement and proof of this important lemma.) We illustrate by computing the $n$-level density function for $\mathrm{U}(N)$. Note that

$$
\lim _{N \rightarrow \infty} \frac{1}{N} J_{N}(\theta / N)=\mathrm{e}(\theta / 2) \frac{\sin \pi \theta}{\pi \theta}
$$

from which it follows easily that

$$
\lim _{N \rightarrow \infty} \frac{1}{N^{n}} \operatorname{det}_{n \times n} J_{N}\left(\theta_{j}-\theta_{k}\right)=\operatorname{det}_{n \times n} K_{0}\left(\theta_{1}, \ldots, \theta_{n}\right)
$$


where $K_{\epsilon}$ is defined in section 5.5.

Now let $f(\mathbf{x})=f\left(x_{1}, \ldots, x_{n}\right)$ be a test function. To compute the $n$-level density (compare with section 5.4) we need to evaluate

$$
\lim _{N \rightarrow \infty} \int_{U(N)} \sum_{\substack{\left.i_{1}, \ldots, i_{n}\right) \\ i_{j} \neq i_{k}}} f\left(\hat{\theta}_{i_{1}}, \ldots, \hat{\theta}_{i_{n}}\right) \prod_{j<k}\left|\mathrm{e}\left(\theta_{j}\right)-\mathrm{e}\left(\theta_{j}\right)\right|^{2} d \theta_{1} \ldots d \theta_{N}
$$

By Gaudin's lemma and after using the new expression for the Haar measure and changing variables $\theta_{j} \rightarrow x_{j} / N$, the above is equal to

$$
\begin{aligned}
\lim _{N \rightarrow \infty} & \frac{1}{N^{n}} \int_{[0, N]^{n}} f\left(\theta_{1}, \ldots, \theta_{n}\right) \frac{1}{n !} \operatorname{det}_{n \times n} J_{N}\left(\theta_{j}-\theta_{k}\right) \prod_{j=1}^{n} d \theta_{j} \\
= & \int_{\mathbb{R}^{n}} f\left(x_{1}, \ldots, x_{n}\right) \operatorname{det}_{n \times n} K_{0}\left(x_{1}, \ldots, x_{n}\right) d x
\end{aligned}
$$

so that $W_{U, n}\left(x_{1}, \ldots, x_{n}\right)=\operatorname{det}_{n \times n} K_{0}\left(x_{1}, \ldots, x_{n}\right)$.

\subsection{Formulas for the Density Functions}

- The $n$-level density is $W\left(x_{1}, \ldots, x_{n}\right)=\operatorname{det}_{n \times n} K_{\epsilon}\left(x_{1}, \ldots, x_{n}\right)$ where $K_{\epsilon}\left(x_{1}, \ldots, x_{n}\right)$ is the $n \times n$ matrix with entries

$$
\left(K_{\epsilon}\left(x_{1}, \ldots, x_{n}\right)\right)_{i, j}=\frac{\sin \pi\left(x_{i}-x_{j}\right)}{\pi\left(x_{i}-x_{j}\right)}+\epsilon \frac{\sin \pi\left(x_{i}+x_{j}\right)}{\pi\left(x_{i}+x_{j}\right)}
$$

where $\epsilon=0$ for $\mathrm{U} ; \epsilon=-1$ for $\mathrm{Sp} ; \epsilon=1$ for $\mathrm{O}^{+}$. Also,

$$
W_{O^{-}, n}(\mathbf{x})=\operatorname{det}_{n \times n}\left(K_{-1}(\mathbf{x})\right)+\sum_{m=1}^{n} \delta\left(x_{m}\right) \operatorname{det}_{n-1 \times n-1}\left(K_{-1}^{(m)}(\mathbf{x})\right)
$$

where $\delta$ is the Dirac $\delta$-function and the superscript $m$ denotes that the $m$-th row and $m$-th column have been deleted from $K_{-1}(\mathbf{x})$.

- The lowest zero density is $\nu_{1}(x)$ where

$$
\begin{gathered}
\nu_{1}(x)=-\frac{d}{d x} \prod_{j=0}^{\infty}\left(1-\lambda_{j}(x)\right) \quad \mathrm{U} ; \\
\nu_{1}(x)=-\frac{d}{d x} \prod_{j=0}^{\infty}\left(1-\lambda_{2 j+1}(2 x)\right) \quad \mathrm{Sp} \\
\nu_{1}(x)=-\frac{d}{d x} \prod_{j=0}^{\infty}\left(1-\lambda_{2 j}(2 x)\right) \quad \mathrm{O},
\end{gathered}
$$

where $1 \geq \lambda_{0}(x) \geq \lambda_{1}(x) \ldots$ are the eigenvalues of

$$
\int_{-x / 2}^{x / 2} \frac{\sin \pi(t-u)}{\pi(t-u)} f(u) d u=\lambda(x) f(t)
$$


- The consecutive spacing density is

$$
\mu(x)=\prod_{j=0}^{\infty}\left(1-\lambda_{j}(x)\right) .
$$

- The $n$-correlation density, $V\left(x_{1}, \ldots, x_{n}\right)=W_{U, n}\left(x_{1}, \ldots, x_{n}\right)$,

\subsection{The Selberg Integral}

There are many versions of Selberg's integral see $\mathrm{Me}]$; one is as follows. If $\Re \alpha>0, \Re \beta>0, \Re \gamma>-\min \left(\frac{1}{n}, \frac{\Re \alpha}{n-1}, \frac{\Re \beta}{n-1}\right)$, then

$$
\begin{gathered}
\int_{-1}^{1} \ldots \int_{-1}^{1} \prod_{1 \leq i<j \leq N}\left|x_{i}-x_{j}\right|^{2 \gamma} \prod_{j=1}^{n}\left(1-x_{j}\right)^{\alpha-1}\left(1+x_{j}\right)^{\beta-1} d x_{j} \\
=2^{\gamma n(n-1)+n(\alpha+\beta-1)} \prod_{j=0}^{n-1} \frac{\Gamma(1+\gamma+j \gamma) \Gamma(\alpha+j \gamma) \Gamma(\beta+j \gamma)}{\Gamma(1+\gamma) \Gamma(\alpha+\beta+\gamma(n+j-1))}
\end{gathered}
$$

\subsection{Moments of Characteristic Polynomials}

$$
\begin{aligned}
M_{U, N}(s) & =\int_{U(N)}|\operatorname{det}(A-I \exp (-i x))|^{2 s} d A \\
& =\prod_{j=1}^{N} \frac{\Gamma(j) \Gamma(j+2 s)}{\Gamma(j+s)^{2}}, \\
M_{S p, 2 N}(s) & =\int_{S p(2 N)}|\operatorname{det}(A-I)|^{s} d A \\
= & 2^{2 N s} \prod_{j=1}^{N} \frac{\Gamma(1+N+j) \Gamma(1 / 2+s+j+s)}{\Gamma(1 / 2+j) \Gamma(1+s+N+j)}, \\
M_{O, 2 N}(s) & =\int_{O(2 N)}|\operatorname{det}(A-I)|^{s} d A \\
& =2^{N s} \prod_{j=1}^{N} \frac{\Gamma(N+j-1) \Gamma(s+j-1 / 2)}{\Gamma(j-1 / 2) \Gamma(s+j+N-1)} .
\end{aligned}
$$

\section{References}

BH. Brézin, E., Hikami, S.: Characteristic polynomials of random matrices. Preprint

BK. Bogomolny, E. B., Keating, J. P.: Random matrix theory and the Riemann zeros. II. $n$-point correlations. Nonlinearity 9 (1996), no. 4, 911-935 
C. Conrey, J. B.: A note on the fourth power moment of the Riemann zeta-function. Analytic number theory, (Allerton Park, IL, 1995), Progr. Math., 138, Birkhuser Boston, Boston, MA, 1996

CF. Conrey, J. B., Farmer, D. W.: Mean values of L-functions and symmetry, IMRN (to appear), AIM Preprint, www.aimath.org, Vol. 2, no. 8 (1999)

CG1. Conrey, J. B. Ghosh, A.: A conjecture for the sixth power moment of the Riemann zeta-function. IMRN 1998, no. 15, 775-780

CG2. Conrey, J. B., Ghosh, A.: On mean values of the zeta-function. Mathematika 31 (1984), no. 1, 159-161

CGo. Conrey, J. B., Gonek, S. M.: High moments of the Riemann zetafunction. AIM Preprint, www.aimath.org, Vol. 2, no. 2 (1999)

D. Duke, W.: The critical order of vanishing of automorphic L-functions with large level. Invent. Math. 119 (1995), no. 1, 165-174

DFI. Duke, W., Friedlander, J. B., Iwaniec, H.: Bounds for automorphic L-functions. II. Invent. Math. 115 (1994), no. 2, 219-239

F1. Farmer, D. W.: Mean values of $\zeta^{\prime} / \zeta$ and the Gaussian unitary ensemble hypothesis. IMRN (1995), no. 2, 71-82

F2. Farmer, D. W.: Long mollifiers of the Riemann zeta-function. Mathematika 40 (1993), no. 1, 71-87

GV. Goldfeld, D., Viola, C.: Mean values of $L$-functions associated to elliptic, Fermat and other curves at the centre of the critical strip. J. Number Theory 11 (1979), no. 3 S. Chowla Anniversary Issue, 305-320

GG. Goldston, D. A., Gonek, S. M.: Mean value theorems for long Dirichlet polynomials and tails of Dirichlet series. Acta Arith. 84 (1998), no. 2, 155-192

Go1. Gonek, S. M.: On negative moments of the Riemann zeta-function. Mathematika 36 (1989), no. 1, 71-88

Go2. Gonek, S. M.: Mean values of the Riemann zeta function and its derivatives. Invent. Math. 75 (1984), no. 1, 123-141

Gu. Guinand, A. P.: A summation formula in the theory of prime numbers. Proc. London Math. Soc. (2) 50 (1948). 107-119

HL. Hardy, G. H., Littlewood, J. E.: The approximate functional equations for $\zeta(s)$ and $\zeta^{2}(s)$. PLMS (2) 29 (1929), 81-97

H-B2. Heath-Brown, D. R.: The fourth power moment of the Riemann zeta function. Proc. London Math. Soc. (3) 38 (1979), no. 3, 385-422

In. Ingham, A. E.: Mean-value theorems in the theory of the Riemann zeta-function. PLMS (2) 27 (1926), 273-300

I. Iwaniec, H.: On the order of vanishing of modular L-functions at the critical point. Sém. Théor. Nombres Bordeaux (2) 2 (1990), no. 2, 365-376

ILS. Iwaniec, H., Luo, W., Sarnak, P.: Low lying zeros of families of Lfunctions. AIM Preprint, www.aimath.org, Vol. 2, no 1 (1999)

IS1. Iwaniec, H., Sarnak, P.: The non-vanishing of central values of automorphic L-functions and Landau-Siegel zeros. Israel J. Math. (to appear)

IS2. Iwaniec, H., Sarnak, P.: Dirichlet L-functions at the central point. Number theory in progress, Vol. 2 (Zakopane-Kościelisko, 1997), 941-952

J. Jutila, M.: On the mean value of $L\left(\frac{1}{2}, \chi\right)$ for real characters. Analysis 1 (1981), no. 2, 149-161 
KS1. Katz, N. M., Sarnak, P.: Random matrices, Frobenius eigenvalues, and monodromy. AMS Colloquium Publications, 45. AMS, Providence, RI, 1999

KS2. Katz, N. M., Sarnak, P.: Zeroes of zeta functions and symmetry. Bull. Amer. Math. Soc. (N.S.) 36 (1999), no. 1, 1-26

KeSn1. Keating, J., Snaith, N.: Random matrix theory and $\zeta(1 / 2+i t)$. Preprint

KeSn2. Keating, J., Snaith, N.: Random matrix theory and $L$-functions at $s=1 / 2$. Preprint

KMV1. Kowalski, E., Michel, P., VanderKam, J. M.: Mollification of the fourth moment of automorphic L-functions and arithmetic applications. Preprint

KMV2. Kowalski, E., Michel, P., VanderKam, J. M.: Nonvanishing of high derivatives of automorphic L-functions at the center of the critical strip. J. Reine Angew. Math. (to appear)

Me. $\quad$ Mehta, M. L.: Random Matrices, Academic Press, 1991

M1. Montgomery, Hugh L.: Topics in multiplicative number theory. Lecture Notes in Mathematics, Vol. 227. Springer-Verlag, Berlin-New York, 1971

M2. Montgomery, H. L.: The pair correlation of zeros of the zeta function. Analytic number theory (Proc. Sympos. Pure Math., Vol. XXIV, AMS, Providence, R.I., 1973

O. Odlyzko, A. M.: The $10^{20}$ th zero of the Riemann zeta-function and 70 million of its neighbors, AT\&T Bell Laboratories Preprint, (1989)

Oz. $\quad$ Özlük, Ali E.: On the $q$-analogue of the pair correlation conjecture. J. Number Theory 59 (1996), no. 2, 319-351

OS. Özlük, A. E., Snyder, C.: On the distribution of the nontrivial zeros of quadratic $L$-functions close to the real axis. Acta Arith. 91 (1999), no. $3,209-228$

R. Rubinstein, M.: Evidence for a spectral interpretation of the zeros of $L$-functions. PhD thesis, Princeton University (1998)

RS. Rudnick, Z., Sarnak, P.: Zeroes of principal L-functions and random matrix theory, Duke Math. J. 81 (1996), 269-322

S. Selberg, A.: Old and new conjectures and results about a class of Dirichlet series. Collected Papers, Vol. 2, Springer-Verlag, Berlin, 1991

Sh. Shanks, Daniel: Systematic examination of Littlewood's bounds on $L(1, \chi)$. Analytic number theory (Proc. Sympos. Pure Math., Vol. XXIV, St. Louis Univ., St. Louis, Mo., 1972), pp. 267-283. Amer. Math. Soc., Providence, R.I., 1973

So1. Soundararajan, K.: Non-vanishing of quadratic Dirichlet Lfunctions at $\mathrm{s}=1 / 2$. Annals of Math. (to appear). AIM Preprint, www.aimath.org, Vol. 2, no. 3 (1999)

So2. Soundararajan, K.: The horizontal distribution of zeros of $\zeta^{\prime}(s)$. Duke Math. J. 91 (1998), no. 1, 33-59

T. Titchmarsh, E. C.: The Theory of the Riemann Zeta-Function, second edition, Revised by D. R. Heath-Brown. Oxford University Press, Oxford, 1986

W. Weyl, H.: Classical Groups. Princeton University Press, 1946 